\title{
AdQUiSICIÓN DE LOS ATAQUES COMPLEJOS EN ESPAÑOL: ANÁLISIS DESDE LA TEORÍA DE LA OPTIMIDAD*
}

\author{
Pilar Andrea Vivar Vivar**
}

\begin{abstract}
Resumen
Se investigó el desarrollo de la estructura de ataque complejo (AC) en español de Chile desde la Teoría de la Optimidad en 9 hablantes (entre 1; 6 y 2; 8 años). De 354 palabras se detectaron 147 AC del tipo / C +1/ y 207 del tipo / C + / / . Se observó sistematicidad en la identificación de tres etapas: $1^{\text {a }}$. $\mathrm{r}$ reducción del AC a un segmento; $2^{\mathrm{a}}$. $\mathrm{C}$ semivocalización del segundo segmento del AC; $3^{\text {a }}$. 5 producción fiel del AC. Como resultados destaca la baja producción fiel de las sílabas con AC del tipo (CCV), siendo la mayoría de los casos del tipo estructura silábica universal $(\mathrm{CV})$. Se observó un alto porcentaje de mantención del primer elemento en el output de los AC. La jerarquía de restricciones de la $1^{a}$ etapa, ${ }^{*}$ COMPLEXONS reduce los $\mathrm{AC}$, en la $2^{\mathrm{a}}$ etapa *COMPLEXNUC es degradada en la jerarquía, favoreciendo la aparición de semivocales que ocupan el lugar de la líquida o la vibrante. Finalmente, en la $3^{\mathrm{a}}$ etapa, la degradación de *COMPLEXONS avanza desde la semiconsonantización del segundo segmento a la mantención de los dos segmentos del ataque complejo.
\end{abstract}

Palabras clave: Fonología, adquisición fonológica, ataque complejo (AC), teoría de la optimidad (TO), dialecto español de Chile.

\section{ACQUisition of COMPLEX ATTACKS IN SPANish: AnAlysis FROM OPTIMALITY THEORY}

\begin{abstract}
The article studies complex attacks (AC) onset development in 9 speakers of the Chilean variant of Spanish from Optimality Theory (between 6 and 2; 8 of age). 354 words containing onset cluster type (AC) 147 / C $+1 /$ and $207 / C+f /$. Systematic was observed in the identification of tree stages. $1^{\text {a }} \mathbf{f}$ reduction of a firts segment; 2 nd $\boldsymbol{f}$ semivocalización the second segment of the AC; and 3rd. $\int$ faithful production of AC. As results, the low output faithful syllables with $\mathrm{AC}$ type $(\mathrm{CCV})$, the majority of cases of universal syllable structure type (CV). Maintaining a high percentage of the first element in the output of the AC was observed. The hierarchy of constraints of the 1st stage * COMPLEXONS reduces AC in the 2nd stage * COMPLEXNUC is degraded in the hierarchy, favoring the emergence of semivowels that take the place of the liquid or vibrant. Finally, in stage $3,{ }^{*}$ COMPLEXONS degradation semiconsonantización proceeds from the second segment to the maintenance of the two segments of attack complex.
\end{abstract}

Keywords: Phonology, phonological acquisition, attack complex (AC), Optimality Theory (OT), Chile Spanish dialect.

Recibido: 02-03-2014

Aceptado: 04-08-2014

* Este artículo corresponde a la investigación desarrollada por la autora como tesis doctoral.

** Chilena, Doctora en Lingüística, Universidad Católica de Temuco, Temuco, Chile. pvivar@uct.cl 


\section{Introducción}

La investigación empírica sobre el proceso de adquisición fonológica ha sido abordada desde dos perspectivas teóricas fundamentales: el estructuralismo y la fonología natural, llamadas también teorías lineales. Una de las principales críticas que se les hacen a las teorías lineales es que en estos modelos no se consideran aspectos superiores e inferiores al segmento como la mora, la silaba, el pie métrico e inclusive la palabra. Con el surgimiento de las teorías no lineales, la adquisición fonológica recibe importantes aportes, sobre todo desde la fonología métrica y prosódica, en las cuales los segmentos son tratados de manera muy diferente según la posición que ocupen dentro de la sílaba. A principios de la década de los noventa, se configura una nueva teoría que se introduce con bastante fuerza en todos los campos de descripción y explicación fonológica, la Teoría de la Optimidad (Prince y Smolensky, 1993). La fortaleza de este planteamiento para el estudio del habla infantil radica en que da cuenta de una adecuada relación entre los datos empíricos y la teoría, ya que permite realizar hipótesis muy precisas y también comprobarlas. La marcadez es fundamental para comprender, desde la TO, el proceso de adquisición, lo que le entrega además un fuerte poder explicativo y comparativo. Todos estos argumentos sirven de base para proponer esta teoría como la indicada para una explicación y descripción fonológica a partir de datos reales. Su perspectiva no lineal, además, permite la descripción de elementos superiores e inferiores al segmento, con la posibilidad de observar al mismo tiempo aspectos segmentales y prosódicos. A pesar de la amplia gama de descripciones que existen sobre adquisición y desempeño fonológico infantil, en la actualidad, siguen considerándose en Chile como guía las perspectivas articulatorias y de procesos fonológicos de simplificación (PFS), las cuales no explican el funcionamiento fonológico que subyace a la evidencia articulatoria misma. En Chile, además, no existen investigaciones sobre adquisición fonológica en menores de tres años, lo cual es una falencia importante en este campo de investigación, ya que los principales avances fonológicos se producen previo a esta edad, ya que sobre los tres años de edad los sujetos ya deberían tener adquirido casi el $80 \%$ de su repertorio fonético. Esto ya ha sido evidenciado para el dialecto español de Chile (Vivar \& León, 2009). En el español de Chile ya se ha utilizado esta perspectiva en el análisis de los grupos consonánticos de ataques complejos. Finalmente, es necesario comenzar la investigación fonológica abordando aspectos 
relativos a la estructura de la sílaba, específicamente a la escala de sonancia o sonicidad. Para comprender el término de sonancia, la sílaba debe ser entendida como un conjunto de segmentos agrupados alrededor de una cumbre o pico de sonancia, de tal modo que los segmentos más cercanos al núcleo tienen un índice de sonancia que nunca es menor al de los más alejados (Núñez y Morales-Front, 1999). En definitiva, la sonancia de un segmento se define en términos de facilidad de percepción y de grado de apertura del tracto vocal. Así, los segmentos pueden ordenarse de mayor a menor grado de sonancia a lo largo de una Jerarquía de Sonancia (Clements, 1990).

\section{Marco teórico}

\section{Fundamentos teóricos de la Teoría de la Optimidad (TO):}

En 1993, Alan Prince y Paul Smolensky formulan en su trabajo Optimality Theory: Constraint interaction in generative grammar, una teoría fonológica que denominaron Teoría de la Optimidad. La fortaleza de esta teoría se debe a que su formalización es demostrable en datos reales, lo cual viene a suplir una de las principales críticas a la teoría generativa: su alto grado de abstracción. En términos generales, lo que se plantea en la TO es que, a partir de una forma subyacente, son posibles las formas superficiales. La relación entre estos inputs y outputs es evaluada por un algoritmo constituido por un conjunto de condiciones de buena-formación o restricciones, el cual selecciona entre todos los posibles candidatos al verdadero (óptimo) (D'Introno et al., 1995). La propuesta principal de la TO está en la aplicación de las restricciones como elementos que permiten llegar a las formas superficiales. Tales restricciones tienen tanto una base fonética como tipológica. Por ejemplo, en el plano silábico existen dos restriccios, ONSET --> toda sílaba debe tener un ataque silábico y ${ }^{*}$ COMPLEX --> no se permite la existencia de ataques ni de codas silábicas complejas. En el caso de ONSET, es sabido que la existencia de ataque silábico es obligatoria en muchas lenguas y opcional en otras. Lo anterior tiene una explicación fonética, ya que la presencia de los patrones de transición entre consonante y vocal (CV) maximizan la perceptibidad del núcleo silábico (Cutillas, 2003).

Para el caso de la restricción *COMPLEX, se aplica la misma lógica, ya que la mayoría de las lenguas no organizan su estructura silábica alrededor de ataques ni codas complejos, lo que podría demostrar que 
la estructura de sílaba que determina la superficie es CV. Por ejemplo, una lengua sólo con estructura $\mathrm{CV}$ tendrá secuencias como $\mathrm{CV}, \mathrm{CVCV}$, CVCVCV, etc., pero nunca estructuras como VCV, CVC, CVCVC, etc. (Archangeli, 1999). Tanto la evidencia tipológica como fonética se relacionan ineludiblemente con un aspecto de larga tradición lingüística: la marcadez o teoría del marcado. Desde esta perspectiva, los elementos no marcados serán aquellos que concuerden con las tendencias generales presentes en todas las lenguas. En resumen, existen dos tipos de restricciones en la TO: las restricciones de fidelidad, requieren que las formas del output se asemejen a las formas del input y las restricciones de marcadez, que requieren que las formas del output sean no marcadas de acuerdo con los principios universales de marcadez.

Las restricciones de fidelidad regulan la relación entre el input y el output impidiendo que el output se aleje del input. Toda infracción que es cometida contra la forma del input ha de estar motivada por alguna restricción de marcadez, que al tener que satisfacerse obligatoriamente provoca la infracción. Las restricciones de marcadez demandan la aparición de estructuras no marcadas, como ONSET, o prohíben la aparición de elementos marcados como CODA y COMPLEX. Estas restricciones provocan la preferencia por sílabas $\mathrm{CV}$; en consecuencia, cualquier otro tipo silábico infringe, por lo menos, una de estas restricciones (Archangeli, 1999).

La universalidad de las restricciones no funciona de la misma forma en cada lengua, lo cual introduce un aspecto radical en el comportamiento de las restricciones en la TO: su infraccionabilidad. Dicha infraccionabilidad se relaciona con la interacción entre las restricciones de marcadez y las de fidelidad. Esto tiene como consecuencia la preferencia hacia distintos tipos silábicos en la tipología general de las lenguas. La relación entre las restricciones, la forma subyacente y la evaluación de las formas superficiales queda reflejada en la tabla estándar utilizada en la TO (1):

\begin{tabular}{|c|c|c|}
\hline /candidato/ & Restricción $\mathrm{X}$ & Restricción $\mathrm{Y}$ \\
\hline $\boldsymbol{\sigma} \quad$ [candidato $\mathrm{a}]$ & & \\
\hline [candidato $\mathrm{b}]$ & ${ }^{*}$ \\
\hline
\end{tabular}


Las restricciones se ubican en las columnas, mientras que los posibles candidatos, en las filas; ellos serán evaluados de izquierda a derecha en función de las restricciones. En términos generales, la tabla muestra una simple relación de dominio entre una restricción de mayor rango sobre otra de menor. Para el caso de esta ilustración, diremos que: RESTRICCIÓN X > RESTRICCIÓN Y, es decir, la restricción " $X$ " se encuentra en una relación de dominio sobre la restricción " $Y$ ". En consecuencia, es el [candidato a] el que será elegido como óptimo, debido a que el [candidato b] incurre en una infracción fatal (*!) al infringir la restricción de mayor rango.

Algunas de las restricciones de marcadez utilizadas en el análisis de la estructura silábica son:

(2) ONSET

${ }^{*}$ CODA

: las sílabas deben tener un ataque u onset.

: las sílabas no deben tener coda.

*COMPLEX-ONSET : los ataques no deben ser complejas.

*VOICED-CODA : : las codas silábicas serán preferentemente sordas.

Para que los outputs de estructura silábica se produzcan fielmente en una determinada lengua, las restricciones de marcadez en (2) deberán entrar en conflicto y ser dominadas por las restricciones de fidelidad (3):

(3) MAXI-O : todos los segmentos del input deben aparecer en el output (contra las elisiones).

DEP I-O : todos los segmentos del output deben estar también en el input (contra la epéntesis).

LINEARITY : el orden de los segmentos en el input debe mantenerse en el output (contra la metátesis).

FAITH : todas las restricciones de fidelidad.

El rango superior de FAITH (fidelidad) queda manifestado en tanto las restricciones de fidelidad dominan a las restricciones de marcadez: FAITH $>>$ ONSET $>>$ *CODA:

(4)

\begin{tabular}{|c|c|c|c|}
\hline Ca.la & FAITH & ONSET & ${ }^{\circ}$ CODA \\
\hline a. $\quad \sigma \quad[a . l a]$ & & $\bullet$ & \\
\hline b. $\quad[$ ta.la] & $* !$ & & \\
\hline c. $\quad[\mathrm{al}$ a] & & $\cdots !$ & $\cdot$ \\
\hline $\begin{array}{ll}\text { d. } & \text { [la] }\end{array}$ & $\bullet 1$ & & \\
\hline
\end{tabular}


En (4), los candidatos con epéntesis (4b) y elisión (4d) transgreden la restricción de fidelidad, ya que alteran la representación subyacente, ya sea por la inserción (4b) o por la elisión (4d) de segmentos. En relación a (4a) y (4c), se produce una infracción de la restricción ONSET al no tener ataques silábicos. No obstante, el candidato (4a) sólo incurre en una infracción de ONSET; mientras que (4c) suma a la doble infracción de ONSET, la de ${ }^{*} \mathrm{CODA}$, lo que lo convierte en un candidato menos óptimo que ['a.la] (Colina, 2006). Aunque en relación con las restricciones el candidato (4a) no es perfecto, es el que infringe la menor cantidad de restricciones.

\section{Adquisición fonológica desde la teoría de la optimidad:}

La investigación en adquisición fonológica ha cobrado un importante valor en la TO, ya que ésta permite, por un lado, conocer cómo se comportan las jerarquías de restricciones en cada lengua; y, por otro lado, analizar las similitudes y diferencias entre las gramáticas observando las mismas restricciones. En cuanto a la relación entre la gramática infantil y la gramática adulta, la fonología natural (Stampe, 1969) plantea que al intentar reproducir el habla adulta se manifiesta en el habla infantil un determinado repertorio de PFS innatos. Desde esta perspectiva, el proceso de adquisición de la lengua materna habrá concluido cuando hayan desaparecido dichos procesos. Por el contrario, en la TO la idea básica es que el niño nace con un conjunto de restricciones que necesita (re)jerarquizar y, a diferencia de la supresión de reglas (o procesos), las restricciones siguen presentes en la gramática.

En las investigaciones sobre la fonología infantil, uno de los cambios más fundamentales que produjo la TO, en relación con las teorías de adquisición previas, es que ha restablecido la continuidad entre la gramática adulta y las que se encuentran en desarrollo, al considerar que ambas están compuestas de las mismas unidades de organización y principios (Boersma \& Levelt, 2003). Las restricciones usadas en el lenguaje adulto deberían, por lo tanto, ser adecuadas para dar cuenta de la fonología infantil sin atribuirle al niño otros niveles representacionales o más reglas que las que el adulto tiene (Gnanadesikan, 2004).

En la investigación y descripción teórica de la fonología infantil, la TO utiliza dos familias principales de restricciones: las de fidelidad (F) 
y las de marcadez (M) (o estructura). El supuesto básico es que en los primeros estadios de adquisición todas las restricciones de marcadez (segmentales, silábicas y prosódicas) superan a las de fidelidad. El proceso de adquisición consiste entonces en una rejerarquización gradual entre las restricciones de marcadez y las de fidelidad, de manera que las primeras van perdiendo fuerza en favor de estas últimas (Gnanadesikan, 2004; Boersma \& Levelt, 2003; Levelt, Schiller \& Levelt, 1999/2000). Por ejemplo, algunas restricciones de marcadez relacionadas con la estructura silábica, tales como ONSET, *CODA y *COMPLEX, inicialmente superan a las restricciones de fidelidad. En consecuencia, la estructura CV se manifiesta como la única posible en los outputs de la gramática infantil (Levelt, Schiller \& Levelt, 1999/2000). En la teoría de la optimidad, uno de los temas centrales es el surgimiento de las estructuras no marcadas durante el proceso de adquisición fonológica. Básicamente, se plantea que los outputs en las primeras etapas de la adquisición serán estructuralmente no marcados y, por lo tanto, no fieles al input esperado. A medida que avanza el proceso de rejerarquización de las restricciones, las producciones comenzarán a ser cada vez más marcadas, si la lengua que el niño adquiere así lo exige. Finalmente, la rejerarquización se detendrá cuando los aprendices de una lengua ya no detecten diferencias entre su output y el lenguaje que los rodea (Levelt, Schiller \& Levelt, 1999/2000). 


\section{Marco Metodológico}

\section{Sujetos}

La muestra está constituida por 9 sujetos distribuidos en 4 grupos de edad. Cada grupo de edad está compuesto de 2 a 3 sujetos, los cuales han sido grabados durante aproximadamente cuatro meses con una frecuencia que fluctúa entre dos a tres veces al mes. La distribución de los niños en los grupos de edad puede observarse en (5):

(5) Distribución de la muestra:

\begin{tabular}{lccc}
\hline \multicolumn{1}{c}{ Grupos } & Edades & $\mathbf{N}^{\circ}$ sujetos & $\mathbf{N}^{\circ}$ de grabaciones \\
\hline Grupo 1 & $1 ; 6-1 ; 8$ & 3 & 34 \\
\hline Grupo 2 & $1 ; 9-1 ; 11$ & 2 & 26 \\
\hline Grupo 3 & $2 ; 0-2 ; 3$ & 2 & 20 \\
\hline Grupo 4 & $2 ; 4-2 ; 8$ & 2 & 14 \\
\hline Total & & 9 & 94 \\
\hline
\end{tabular}

Toma de la muestra:

La mayoría de los sujetos fueron evaluados en sus mismos hogares y en presencia de sus padres o familiares más cercanos con el fin de facilitar su interacción social y comunicativa. Cada sesión está almacenada en formato WAV en una base de datos general de todos los sujetos. En dicha base de datos, es codificada la edad del sujeto al momento de la grabación y fecha de realización de la misma. Posteriormente, cada sesión es transcrita fonéticamente (según las fuentes del Alfabeto Fonético Internacional (AFI; en inglés, International Phonetic Alphabet: IPA) e ingresadas al programa EXMARaLDA ${ }^{1}$. Cada sesión transcrita en EXMARaLDA especifica en distintas columnas lo siguiente: a) número de la emisión y momento en que ésta se produce (min. y seg.); b) si la emisión es espontánea o imitada; c) transcripción fonética y ortográfica; d) comentarios que el transcriptor estime conveniente. El programa EXMARaLDA permite también ingresar más información sobre la entrevista, por ejemplo, las intervenciones de los evaluadores.

1 Sonderforschungsbereich für Mehrsprachigkeit 538 (Centro de Investigación Sobre el Multilingüismo 538, Hamburgo). 
Análisis de los datos:

Se transcribieron un total de 354 palabras que contenían ataques complejos (ACs) de los cuales 147 son / C+1/ y $207 / \mathrm{C}+\boldsymbol{\Gamma} /$. Es muy importante mencionar el hecho de que la corta edad de los sujetos impide aplicar una prueba estándar de nombrado, con las cuales se obtienen datos equilibrados El manejo del léxico entre los 1; 6 a los 2; 8 años de edad es reducido, por lo que la investigadora tuvo que acudir a entrevistas espontáneas para elicitar los ataques complejos en los niños en función de su experiencia de mundo y almacén léxico. Para analizar los datos, se clasificaron los distintos desajustes cometidos por los sujetos de acuerdo a los siguientes parámetros:

a) Mantención del primer elemento $\left(\mathrm{C}_{1}\right)$ : el sujeto mantiene el primer elemento en el output de manera fiel, por ejemplo en ['ban.ko] por blanco. También se considera mantención del primer elemento a las consonantes de baja sonancia, por ejemplo ['paך.ko] por blanco.

b) Mantención del segundo elemento $\left(\mathrm{C}_{2}\right)$ : el sujeto mantiene el segundo elemento de manera fiel, por ejemplo, en ['lan.ko] por blanco. También se considera mantención del segundo elemento a las consonantes sonorantes, aunque difieran de la del input, por ejemplo [' raף.ko] por blanco.

c) Mantención de ambas posiciones con semivocalización de la segunda (C1CG): por ejemplo en ['bjan.ko] por blanco.

d) Ausencia de ataque (ØV): por ejemplo, en ['an.ko] por blanco.

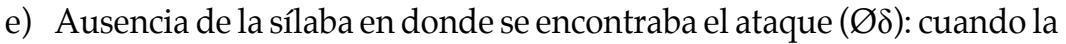
sílaba completa en que se encontraba el ataque complejo es eliminada, por ejemplo, en ['ti.ja] por frutilla. En estos casos, el ataque complejo no es analizado.

\section{Resultados}

Se presentan los distintos estadios de adquisición que evidencian los sujetos, analizados desde la Teoría de la Optimidad. El objetivo es conocer qué restricciones están operando durante el proceso de adquisición de los ACs. Los ACs han sido organizados para su análisis de acuerdo al punto de articulación (PA) desde la zona anterior a la posterior. Por lo tanto, los primeros ataques complejos analizados son los compuestos por bilabial, 
seguidos de labiodental, coronal (sólo en el caso de / obstruyente+ $\mathbf{\Upsilon} /$ ) y finalmente, dorsal.

\section{Estadio 1: Mantención de $\mathrm{C}_{1}$ en /C+1/}

En la primera etapa, la mayoría de los niños mantiene la primera consonante del AC $\left(C_{1}\right)$. Esto sugiere que en este momento de su desarrollo están operando dos restricciones de marcadez, una que prohíbe los ataques complejos: ${ }^{*}$ COMPLEX, y otra que prohíbe los ataques con lateral: *L-ONSET. A continuación se presenta el análisis con las emisiones infantiles divididas según el PA de la obstruyente.

a) Mantención de $\mathrm{C}_{1}$ : Bilabiales

Algunos ejemplos de las emisiones infantiles en este tipo de AC se presentan en (6):

(6) Mantención de C1 en bilabiales $+/ 1 /$ :

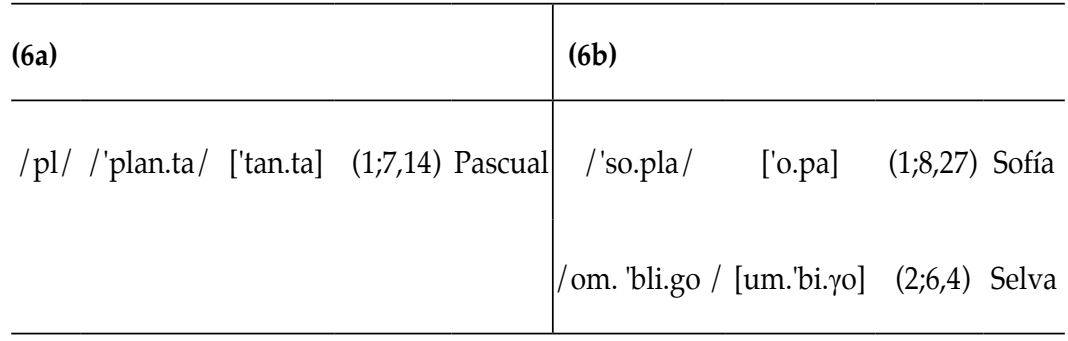

En los ataques complejos de obstruyente labial seguida de líquida lateral, se evidencian dos tipos de fenómeno en la mantención de la C1. En la mayoría de los casos se mantiene la consonante bilabial fielmente (6b); mientras que, en otros casos en que el ataque de la segunda sílaba es coronal, la bilabial inicial es sustituida por una consonante coronal (6a). En el primer caso, las restricciones que están operando son: *COMPLEX y *L-ONSET; esto quiere decir que el candidato óptimo será un ataque simple constituido por una obstruyente bilabial. El orden de las restricciones se muestra por medio de Tablas (7), que en la línea superior reflejan la jerarquía de restricciones de izquierda a derecha, de más a menos dominancia, y en la columna de la izquierda muestra la palabra de la lengua adulta y algunos de los candidatos: 


\begin{tabular}{rccc}
\hline /'pla.to/ & ${ }^{*}$ COMPLEX & ${ }^{*}$ L-ONSET & MAX \\
\hline a. ['pla.to] & ${ }^{*} !$ & ${ }^{*}$ & \\
\hline अ b. ['pa.to] & & & ${ }^{*}$ \\
\hline c. [la.to] & & ${ }^{*} !$ & ${ }^{*}$ \\
\hline
\end{tabular}

En (7a), se infringe *COMPLEX, que es una restricción que no permite AC en el output, mientras que en (7c) se infringe *L-ONSET, que prohíbe la aparición de laterales en el ataque. Por lo tanto, el candidato óptimo en este momento de la adquisición es $(7 \mathrm{~b})$, lo cual, según la terminología de optimidad queda señalizado con el símbolo ३3. Por su parte, en el caso de las emisiones observadas en (6a), está operando una restricción de marcadez que actúa en contra de los ataques bilabiales iniciales, si la palabra contiene también un ataque coronal. En este caso, una restricción de concordancia del rasgo coronal (8) impide la aparición de labial seguida de coronal:

(8) CONC(COR): CONCORDANCIA del rasgo CORONAL conduce a la asimilación a distancia ${ }^{2}$ (o armonía), en el caso de que la palabra contenga otra consonante coronal; equivale al inglés AGREE.

El orden de las restricciones queda reflejado en (9):

(9) *COMPLEX >> *L-ONSET, CONC(COR) >> MAX

\begin{tabular}{ccccc}
\hline /'pla.to/ & ${ }^{*}$ COMPLEX & ${ }^{*}$ L-ONSET & CON(COR) & MAX \\
\hline a. ['pla.to] & ${ }^{*} !$ & & ${ }^{*}$ & \\
\hline b. ['pa.to] & & & ${ }^{*} !$ & ${ }^{*}$ \\
\hline c. [la.to] & & ${ }^{*} !$ & & ${ }^{*}$ \\
\hline \% d. [do.ta] & & & & ${ }^{*}$ \\
\hline
\end{tabular}

El output (9a) infringe la restricción de marcadez *COMPLEX, que prohíbe grupos consonánticos en el output, mientras que (9b) infringe la restricción $\mathrm{CONC}(\mathrm{COR})$, la cual opera a favor de la asimilación de la coronal; finalmente (9c) infringe la restricción de marcadez *L-ONSET,

2 "A distancia", porque entre las dos coronales hay una vocal, de manera que las dos coronales no están una al lado de la otra; los casos de asimilación inmediata son por ej.: campo, diente, tango, en que la nasal adopta el punto de articulación de la obstruyente contigua. 
que opera en contra de la aparición de ataques laterales en el output. La línea punteada entre las restricciones *L-ONSET y CONC(COR) indica que no hay dominio de una restricción sobre otra. La forma óptima es (9d), que sólo infringe la última restricción de la jerarquía: la restricción de fidelidad MAX. Nótese que la diferencia entre las dos jerarquías de restricciones, entre (7) y (9), consiste en la presencia de CONC(COR) solamente en (9). Este tipo de restricción limita los puntos de articulación de las palabras infantiles a uno solo, lo cual es frecuente en las primeras etapas del desarrollo fonológico infantil. Por tanto, la jerarquía de (7), en la que CONC(COR) ya no opera, es propia de una etapa más avanzada que la de (9). Lo anterior también se ve reflejado en las edades de las emisiones infantiles en que las emisiones en (6a) corresponden a edades inferiores a (6b).

b) Mantención de $C_{1}$ : labiodental

En el $\mathrm{AC} / \mathrm{fl} /$, la mayoría de los niños mantiene la $\mathrm{C}_{1}$ mediante dos formas: mediante una obstruyente coronal (10a) o mediante una obstruyente bilabial (10b). En ambos casos, están operando tres restricciones de marcadez, una que prohíbe los ACs, *COMPLEX, y otras dos que prohíben los ataques con lateral o con fricativa: *L-ONSET y ${ }^{*}$ F-ONSET, respectivamente:

(10) Mantención de $C_{1}$ en labiodental + /1/:

\begin{tabular}{|c|c|c|c|c|c|c|}
\hline \multicolumn{4}{|l|}{ (10a) } & \multicolumn{3}{|l|}{$(10 b)$} \\
\hline /fl/ /'flo.res / & ['to.te $\left.{ }^{\mathrm{h}}\right]$ & $(1 ; 9,13)$ & Sofía & /'u.na.flor/ ['u.na.flof] & $(2 ; 2)$ & Seba G. \\
\hline /'flo.res / & ['te.te] & $(1 ; 7,2)$ & Iko & / flor/ & $(2 ; 3,13$ & ) Seba G. \\
\hline
\end{tabular}

Por lo tanto, en este estadio de los ACs con labiodental más lateral, el candidato óptimo es un ataque simple constituido por la consonante coronal en unos casos, y oclusiva bilabial en otros. La aparición de la obstruyente labial (10b) indica fidelidad al PA labial de la obstruyente del input (/f/), de lo cual puede darse cuenta mediante la restricción de fidelidad siguiente: 
(6) IDENT(LABIAL): Manténgase el PA labial del input.

En estos casos, el análisis conduce a la siguiente jerarquía de restricciones:

(11) ${ }^{*}$ COMPLEX $>>{ }^{*}$ L-ONSET $>>{ }^{*}$ F-ONSET $>>$ IDENT(LABIAL) $>>$ MAX

\begin{tabular}{cccccc}
\hline$/$ flo $/$ & ${ }^{*}$ COMPLEX & ${ }^{*}$ L-ONSET & ${ }^{*}$ F-ONSET & IDENT(LAB) & MAX \\
\hline a. [flor] & ${ }^{*} !$ & ${ }^{*}$ & ${ }^{*}$ & & \\
\hline b. [for] & & & ${ }^{*} !$ & & ${ }^{*}$ \\
\hline c. [lor] & & ${ }^{*} !$ & & & ${ }^{*}$ \\
\hline a d. [po] & & & & ${ }^{*}$ \\
\hline e. [to] & & & & ${ }^{*} !$ & ${ }^{*}$ \\
\hline
\end{tabular}

El output (11a) es eliminado porque infringe la restricción de marcadez *COMPLEX, la cual opera en contra de la aparición del AC. Por su parte, los outputs (11b) y (11c) infringen restricciones de marcadez que operan en contra de la aparición de fricativas y laterales, respectivamente. La restricción que permite distinguir entre los outputs (11d) y (11e) es IDENT(LAB) la cual opera a favor de la mantención del punto de articulación (en este caso, labial); en consecuencia (11d) se configura como el candidato óptimo.

Por otro lado, la mayoría de los casos en que la obstruyente adopta el PA coronal son palabras bisilábicas, con un ataque coronal en la segunda sílaba (10a). Esto parece indicar que se trata de la restricción de CONCORDANCIA introducida más arriba (8). Es decir, estos casos infringen IDENT(LABIAL) y respetan CONC(COR). Sólo Selva (10) reduce el $\mathrm{AC} / \mathrm{fl} /$ a la fricativa /f/, lo cual indica que la restricción en contra de los ataques constituidos por / f/ ha descendido en su jerarquía de restricciones.

c) Mantención de $C_{1}$ : dorsales

$\mathrm{Al}$ igual que los ataques complejos con bilabiales, en aquellos con dorsales se producen dos tipos de mantención del primer elemento: de manera fiel (12a) y por medio de consonante coronal o labial (12b).

(12) Mantención de $C_{1}$ en dorsales $+/ 1 /$ : 


\begin{tabular}{llll}
\hline (12a) & $\mathbf{( 1 2 b )}$ \\
\hline / kl/ / bi.si.'kle.ta/ [i.ji.'ke.ta] & $(2 ; 0,27)$ Agustina & /bi.si.'kle.ta/ [9.'te.ta] (1;11) Iko \\
/gl/ /'glo.bo/ ['go.ba] & $(1 ; 8,6) \quad$ Iko & /'glo.bo/ ['Bo.va] $(1 ; 7,6)$ Sofía \\
\hline
\end{tabular}

${ }^{*}$ COMPLEX, ${ }^{*} \mathrm{~L}-\mathrm{ONSET}$. Lo anterior quiere decir que el candidato óptimo será un ataque simple constituido por una consonante dorsal (13): (13) * COMPLEX $>>{ }^{*}$ L-ONSET $>>$ MAX

\begin{tabular}{cccc}
\hline /bi.si.'kle.ta/ & ${ }^{*}$ COMPLEX & ${ }^{*}$ L-ONSET & MAX \\
\hline a. [(bi.si)'kle.ta] & $* !$ & ${ }^{*}$ & \\
\hline [3b. [(bi.si)'ke.ta] & & & ${ }^{*}$ \\
\hline c. [(bi.si)'le.ta] & ${ }^{*} !$ & ${ }^{*}$ & ${ }^{*}$ \\
\hline
\end{tabular}

En el segundo grupo de ejemplos en (12b), los ataques / $\mathrm{kl} /$ son sustituidos por consonantes coronales, lo cual supone que en este tipo de emisiones opera la restricción CONC(COR) (14). Por su parte, los ataques /gl/ son sustituidos por consonantes labiales (12b), lo cual, al igual que en el caso de los ACs $/ \mathrm{kl} /$, supone que en este tipo de emisiones opera una restricción de concordancia, en este caso, con el punto labial CONC(LAB). Recuérdese que esta concordancia es con el punto de articulación de la consonante de la segunda sílaba de globo.

(14) ${ }^{*}$ COMPLEX $>>{ }^{*} \mathrm{~L}-\mathrm{ONS}>>\mathrm{CONC}(\mathrm{COR})>>\operatorname{IDENT}(\mathrm{DOR})>>\mathrm{MAX}^{3}$

\begin{tabular}{|c|c|c|c|c|c|}
\hline /bi.si.'kle.ta/ & ${ }^{*}$ COMPLEX & ${ }^{*} \mathrm{~L}-\mathrm{ONS}$ & CONC(COR) IDEN & $\mathrm{JT}(\mathrm{DOR})$ & MAX \\
\hline a. [(bi.si)'kle.ta] & *! & * & * & & \\
\hline b. [(bi.si)'te.ta] & & & & * & * \\
\hline c. [(bi.si)'le.ta] & & ${ }^{*} !$ & & & * \\
\hline d. [(bi.si)'ke.ta] & & & *! & & * \\
\hline
\end{tabular}

3 El análisis solo incluye el segundo pie métrico de la palabra en cuestión, ya que el primer pie no es pertinente al análisis y por eso se encuentra entre paréntesis. 
El output (14a) comete una infracción mayor contra *COMPLEX, por su parte $(14 \mathrm{c})$ infringe la restricción de marcadez * L-ONS, que prohíbe ataque con lateral, mientras que (14d), al mantener la dorsal del input, infringe la restricción de concordancia.

\section{Estadio 1: Mantención de C1 en /C+r/}

$\mathrm{Al}$ igual que en $/ \mathrm{C}+1 /$, en esta primera etapa la mayoría de los niños mantienen la $\mathrm{C} 1$. Lo anterior sugiere que en este momento de su desarrollo están operando dos restricciones de marcadez, una que prohíbe los ataques complejos ${ }^{*}$ COMPLEX y la otra que aboga por la minimización de la sonancia en los ataques, prohibiendo los ataques con sonorante: *SON-ONSET. A continuación, se presenta el análisis con las emisiones infantiles divididas según el PA:

a) Mantención de $\mathrm{C}_{1}$ : bilabiales

(15) Mantención de $C_{1}$ : bilabiales $+/$ / /:

\section{$\mathrm{CCV} \rightarrow \mathrm{C}_{1}$ bilabiales}

$\begin{array}{lllll}\text { /br } / & \text { /'bra.bo/ } & {[\text { 'ba.bo] }} & (1 ; 6,25) & \text { Pascual } \\ / \mathrm{pr} / & \text { /a.pre.'tan.do/ } & {[\text { [Be.'tan.to] }} & (2 ; 4,28) & \text { Selva }\end{array}$

En este tipo de ataque complejo, se mantiene la consonante bilabial fielmente, por lo que las restricciones que están operando son: ${ }^{*}$ COMPLEX y *SON-ONSET. En consecuencia, el candidato óptimo será un ataque simple, constituido por una consonante bilabial. Lo anterior se puede observar en (16):

(16) * ${ }^{*}$ COMPLEX >> * SON-ONSET $>$ MAX

\begin{tabular}{|c|c|c|c|}
\hline /'bra.bo/ & ${ }^{*}$ COMPLEX & *SON-ONS & MAX \\
\hline a. ['b ra. $\beta$ o] & $*$ & * & \\
\hline अ b. ['ba.ßo] & & & * \\
\hline c. ['ra.ßo] & & *! & * \\
\hline
\end{tabular}


b) Mantención de $\mathrm{C}_{1}$ : labiodental

En el ataque complejo / fr/, los niños evidencian dos variantes en la mantención de C1, la primera en forma de una obstruyente coronal (17a) y la segunda con la mantención fiel del primer elemento, la consonante labiodental (17b):

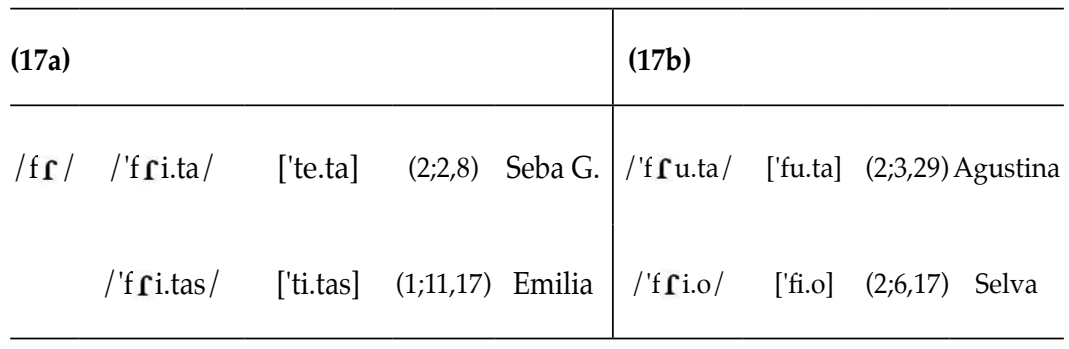

Al igual que en el ataque / fl/, la mantención del primer elemento se hace de manera fiel o infiel a la fricativa. Los casos en que se hace de manera infiel son debidos a la restricción CONC(COR), la cual da cuenta de la no coincidencia entre la consonante del output y la del input:

(18) *COMPLEX >> *SON-ONS $>>$ CONC $(C O R)>>$ MAX

\begin{tabular}{ccccc}
\hline /'fri.ta/ & ${ }^{*}$ COMPLEX & ${ }^{*}$ SON-ONS & CONC(COR) & MAX \\
\hline a. ['fri.ta] & $*$ ! & $*$ & $*$ & \\
\hline b. ['fi.ta] & & & $*$ ! & $*$ \\
\hline c. ['ri.ta] & & $*$ ! & & $*$ \\
\hline (3. ['te.ta] & & & & $*$ \\
\hline
\end{tabular}

El output (18a) infringe la restricción de marcadez *COMPLEX, mientras que (18b) infringe la restricción CONC(COR), la cual opera a favor de la asimilación de la consonante inicial al punto de articulación del ataque siguiente. Finalmente, (18c) infringe la restricción de marcadez *SON-ONS, que opera en contra de la aparición de ataques sonorantes en el output. El candidato óptimo, por lo tanto, es el output ['te.ta] por frita, que respeta todas las restricciones, excepto MAX.En los casos de Agustina y Selva, se produjo el ataque complejo / $\mathrm{f} \boldsymbol{\mathbf { r }} /$ reducido fielmente a la fricativa: 
(19) *COMPLEX >> *SON-ONS >>MAX

\begin{tabular}{cccc}
\hline$/$ 'fru.ta/ & ${ }^{*}$ COMPLEX & ${ }^{*}$ SON-ONS & MAX \\
\hline a. ['fru.ta] & ${ }^{*}$ ! & & \\
\hline a b. ['fu.ta] & & & ${ }^{*}$ \\
\hline c. ['ru.ta] & & ${ }^{*}$ ! & ${ }^{*}$ \\
\hline
\end{tabular}

c) Mantención de $\mathrm{C}_{1}$ : coronales

Aunque ambas consonantes, [t] y [d], articulatoriamente sólo difieren en el rasgo sonoridad, durante el proceso de adquisición de las mismas, dentro de ACs con vibrante, se comportan de manera distinta con respecto a las restricciones. Este fenómeno puede ser explicado por medio de la frecuencia de aparición (el ataque / $\mathrm{d} \boldsymbol{s}$ / es mucho menos frecuente que / tr / en español), así como también por aspectos articulatorios, ya que los fonemas $[d]$ y $[\boldsymbol{r}$ ] comparten más rasgos que los fonemas $[t]$ y $[\boldsymbol{r}]$, lo que puede redundar en diferencias perceptivas.

Las emisiones producidas para cada tipo de ataque se presentan a continuación:

(20) Mantención de $C_{1}$ : coronales $+/$ / /:

\begin{tabular}{llll}
\hline $\mathrm{C}_{1(t)}$ & & & \\
\hline$/$ tren/ & [te:] & $(1 ; 6,25)$ & Pascual \\
\hline$/$ 'o.tro/ & {$[$ 'o.to] } & $(1 ; 9,11)$ & Iko \\
\hline$/$ es.tre.ja/ & ['te.ja] & $(1 ; 9,25)$ & Iko \\
\hline
\end{tabular}

En los ataques complejos del tipo / t $\mathbf{r}$ /, se evidencia la mantención fiel de la obstruyente coronal. En el caso anterior, las restricciones que están operando son: *COMPLEX y *SON-ONSET; esto quiere decir que el candidato óptimo será un ataque simple consistente en una obstruyente coronal sorda. Lo anterior se puede observar en (21): 
(21) *COMPLEX >> *SON-ONS >> MAX

\begin{tabular}{|c|c|c|c|}
\hline /'o.tro/ & ${ }^{*}$ COMPLEX & *SON-ONS & MAX \\
\hline a. ['o.tro] & ${ }^{*} !$ & & \\
\hline b. ['o.to] & & & * \\
\hline c. ['o. fo] & & $* !$ & * \\
\hline
\end{tabular}

d) Mantención de $\mathrm{C}_{1}$ : dorsales

Al igual que los ataques complejos con lateral, en este tipo de ataque complejo se producen dos tipos de mantención del primer elemento: de manera fiel y por medio de consonantes coronales (23):

(23) Emisiones $\mathrm{C}_{1}$ dorsales $+/ \mathbf{f} /$ :

(23a)

(23b)

/'gra.sias / ['ta.ta] (1;6,25) Pascual /mui.'gran.de/ [mu.'ya.ni] (2;0,27) Agustina

En el primer caso (23a), las consonantes dorsales son sustituidas por consonantes anteriores coronales, lo cual supone que en este tipo de emisiones opera la restricción CONC(COR), que hemos visto más arriba. Es decir, el ataque de la primera sílaba se asimila a distancia al PA del ataque de la sílaba siguiente. La operación de esta restricción pone de manifiesto que en esta etapa temprana de la adquisición, las palabras contienen un solo PA:

(24) *COMPLEX $>>$ *SON-ONSET $>>$ CONC $($ COR $)>>$ *MAX

\begin{tabular}{ccccc}
\hline /'gra.sias/ & ${ }^{*}$ COMPLEX & ${ }^{*}$ SON-ONSET & CONC(COR) & MAX \\
\hline a. ['gra.sjas] & ${ }^{*}$ ! & ${ }^{*}$ & ${ }^{*}$ & \\
\hline *b. ['ta.sja] & & & & ${ }^{*}$ \\
\hline a. ['ga.sjas] & & & ${ }^{*} !$ & ${ }^{*}$ \\
\hline b. ['ra.sjas] & & $* !$ & & ${ }^{*}$ \\
\hline
\end{tabular}


El output (24a) infringe la restricción de marcadez *COMPLEX, que prohíbe grupos consonánticos en el output; mientras que (24c) infringe la restricción $\mathrm{CONC}(\mathrm{COR})$, que penaliza la aparición de más de un PA en la palabra; mientras que $(24 \mathrm{~d})$ infringe la restricción de marcadez *L-ONSET, que opera en contra de la aparición de consonantes líquidas en los ataques del output. Por lo tanto, el candidato óptimo es (24b) con el output ['ta.sja] por gracias. En el segundo caso, las restricciones que están operando son: *COMPLEX y *SON-ONSET (25). Esto quiere decir que el candidato óptimo será un ataque simple, fiel a la obstruyente del ataque meta $(25 b)$ :

*COMPLEX >> *SON-ONSET $>>\operatorname{IDENT}($ DOR) $>>$ MAX

\begin{tabular}{|c|c|c|c|c|}
\hline /'ti.gre/ & ${ }^{*}$ COMPLEX & *SON-ONSET & IDENT(DOR) & MAX \\
\hline a. ['ti. $\gamma r e]$ & ${ }^{*} !$ & & & \\
\hline [अ. ['ti. $\gamma \mathrm{e}]$ & & & & * \\
\hline c. ['ti. re] & & *! & * & * \\
\hline
\end{tabular}

En resumen, en los ACs / $\mathrm{C}+\mathrm{r} /$ durante la primera etapa, todos los sujetos mantienen un elemento del ataque en el output, lo que significa que la restricción *COMPLEX supera a MAX. En el caso de las bilabiales la mantención del primer elemento se hizo de manera fiel. En el caso de la labiodental se manifiestan dos tipos de mantención del primer elemento: la primera de manera infiel y la segunda, fiel. En la primera es introducida la restricción $\mathrm{CONC}(\mathrm{COR})$ que opera a favor de la asimilación del ataque inicial al punto de articulación coronal, si la palabra contiene un segundo ataque coronal. De esta manera se explican emisiones infantiles tales como: ['te.ta] frita. Las obstruyentes coronales evidencian distintas jerarquías entre ellas, según se trate de la consonante sorda o sonora. Los ACs compuestos de dorsal y / $/$ /: en un primer estadio la reducción se lleva a cabo a favor de una obstruyente coronal, si hay otra coronal en el output de la misma palabra. Posteriormente, la obstruyente dorsal del input sobrevive en el output, lo que indica que fidelidad domina a marcadez con respecto al punto de articulación del AC reducido.

\section{Estadio 2: surgimiento de la C1CG en $/ C+1 / y / C+r /$}

Luego de la primera etapa, caracterizada por la reducción de los dos segmentos del AC a un segmento, en la mayoría de los sujetos se observa 
el surgimiento de un segundo elemento semiconsonántico en el output. Dentro de este grupo de niños están quienes manifiestan el surgimiento de la semiconsonante como la segunda y última etapa, y quienes evidencian este comportamiento como una (segunda) etapa previa a la mantención de los dos elementos en el output. Para explicar las emisiones del tipo obstruyente + semiconsonante es necesario revisar las características fonotácticas particulares del español, en donde los diptongos crecientes poseen una frecuencia de aparición considerable (Gili Gaya, 1966). Como consecuencia de lo anterior, los niños hispanohablantes deberían tener experiencias tempranas en el tratamiento de los diptongos, lo cual se ve proyectado también en su tratamiento de los ACs. Algunos ejemplos de la producción de diptongos precedidos de ataques simples y semiconsonantizaciones en los ataques complejos se muestran en (26): (26)

\begin{tabular}{|c|c|c|c|c|c|c|}
\hline /'pa.to/ & ['pja.to] & $(1 ; 6,25)$ & $\rightarrow /$ tren/ & [tje:] & $(1 ; 6,25)$ & Pascual \\
\hline /'a.gua/ & ['a. $\gamma w a]$ & $(1 ; 6,12)$ & $\rightarrow$ /'glo.bo & ['gwa.] & $(1 ; 8,1)$ & Sofía \\
\hline /san.'tia.go/ & [tfan.'tja. $\gamma o]$ & $(2 ; 0,16)$ & $\rightarrow$ / bi.si.'kle.ta/ & [si.'kje.ta] & $(2 ; 2,11)$ & Agustina \\
\hline
\end{tabular}

La observación de las emisiones presentadas (26) indica que la gramática de los sujetos permite la producción de diptongos crecientes, los cuales en la mayoría de los casos, preceden a la producción de los diptongos que sustituyen a un grupo consonántico.

Desde la perspectiva de la optimidad, del mismo modo que tenemos una restricción ${ }^{*}$ COMPLEXONS, que se justifica por la existencia de lenguas que no toleran grupos consonánticos en el ataque, también tenemos una restricción en contra de los núcleos silábicos complejos (27), justificable por la existencia de lenguas que no toleran diptongos:

(27)* COMPLEXNCL: (complejidad del núcleo) penaliza los núcleos silábicos complejos.

De acuerdo a lo anterior, podemos señalar que en las emisiones observadas en (26) los pequeños prefieren infringir *COMPLEXNCL, antes que *COMPLEXONS, por lo que convierten las secuencias de dos consonantes CCV en una obstruyente seguida de la semiconsonante [j] o [w]. La descripción de una palabra, como por ejemplo / t ren/, producida [tjen], se formulará de la manera siguiente en la TO: 
(28) *COMPLEXONS $>>$ MAX $>>{ }^{*}$ COMPLEXNUCL

\begin{tabular}{|c|c|c|c|}
\hline /tren/ & ${ }^{*}$ COMPLEXONS & MAX & *COMPLEXNUCL \\
\hline a. [ten] & & *! & \\
\hline b. [tren] & *! & & \\
\hline 亨 c. [tjen] & & * & \\
\hline
\end{tabular}

Este análisis presupone que en la gramática de los niños en (28), mientras los grupos consonánticos se alojan en el ataque, los diptongos ocupan el núcleo silábico, con lo que la configuración de los diptongos crecientes coincide con la de la lengua adulta. Kehoe, Hilaire-Debove, Demuth \& Lleó (2008) llegan a la conclusión de que en etapas tempranas de la adquisición algunos niños tratan los diptongos crecientes como pertenecientes al ataque, es decir, como grupos consonánticos. Aquí, sin embargo, suponemos que algunos sujetos siguen la trayectoria inversa, al convertir los grupos consonánticos en diptongos. La verdad es que ésta en un área de la lengua que presenta mucha variación entre lenguas distintas y mucha variación individual (ver, por ejemplo, Barlow, 2005). En resumen, durante la segunda etapa se evidencia en la mayoría el surgimiento de un segundo elemento en el núcleo complejo, manifestado mediante la siguiente jerarquía de restricciones: ${ }^{*}$ COMPLEXONS $>>$ MAX $>>$ *OMPLEXNUCL

\section{Estadio 3: surgimiento de $\mathrm{C}_{1} \mathrm{C}_{2}$}

El estadio 3 se caracteriza por el surgimiento del segundo elemento del AC; sin embargo, los pequeños consiguen las formas correctas con distintos ritmos y recorriendo distintas rutas, según el tipo de AC de que se trate y también según las características particulares de su sistema fonológico. La mantención de dos elementos consonánticos en el output se manifiesta de dos formas: en algunos casos se aprecia el paso de los sujetos por las tres etapas $\mathrm{C}_{1}-->\mathrm{C}_{1} \mathrm{C}_{\mathrm{G}}-->\mathrm{C}_{1} \mathrm{C}_{2}$, mientras que en otros casos se evidencia un paso desde $C_{1} /{ }_{2}->C_{1} C_{2}$ : 


\section{Conclusiones}

Se ha propuesto investigar la adquisición de los ataques complejos en niños chilenos. El punto de vista aplicado ha sido el de la Teoría de la Optimidad, según el cual, la gramática que el niño maneja consiste en un conjunto de restricciones, de marcadez y de fidelidad, jerarquizadas de manera específica. Esta teoría propone que al comienzo del proceso de adquisición, las restricciones de marcadez dominan a las de fidelidad. Esta relación se va invirtiendo, según la lengua que el niño adquiere lo exija, es decir, según la dominancia que las restricciones de fidelidad tengan sobre las de marcadez en la lengua a adquirir.

Una de las conclusiones que se obtiene de esta investigación es que durante los primeros estadios de adquisición los niños hablantes del español de Chile tienen una clara tendencia a la reducción de la sílaba CCV a CV. Los aprendices no sólo reducen la estructura silábica, sino que además, mantienen mayoritariamente el primer y menos sonorante elemento del ataque complejo. En consecuencia, esta investigación apoya la postura de que la reducción de los grupos consonánticos (en este caso los ataques complejos) está relacionada con el patrón de sonancia (Chin 1996; Ohala 1999; Barlow 2005a; Pater \& Barlow 2003; Gierut 1999). Durante los primeros estadios de adquisición existe una importante restricción de marcadez responsable de los procesos de armonía consonántica; nos referimos a la restricción CONCORDANCIA, el origen de la cual según Pater (1997) podría relacionarse con las conductas de repetición durante el balbuceo. La interacción de la restricción de marcadez CONCORDANCIA con las restricciones de fidelidad, IDENT(LAB), IDENT(COR) permiten determinar cuál es más importante en la jerarquía de restricciones durante un período determinado de tiempo; en consecuencia, gracias a la infraccionabilidad de las restricciones, en principio, es posible explicar las diferentes variedades de armonía consonántica mediante la interacción de las dos ya mencionadas restricciones. Uno de los aspectos que se ha considerado entre de los más interesantes en los estudios de adquisición de los grupos consonánticos es la relación entre el comportamiento articulatorio de las consonantes en posición aislada y dentro de grupos consonánticos. En este aspecto la respuesta es relativa y dependerá más bien del tipo de consonante de que se trate. En este trabajo, por ejemplo, se concluye que la asimetría que existe entre los grupos / $+1 /$ frente a los grupos $/ \mathrm{C}+\boldsymbol{\Gamma} /$ en la mantención del primer elemento se debe a la 
dificultad articulatoria que presenta la vibrante simple para los pequeños hispanohablantes (Bosch, 2004; González, 1989; Vivar \& León, 2009). En relación con el comportamiento de la jerarquía de restricciones, se puede concluir que ambos tipos de ataques complejos (con lateral y con vibrante) evidencian bastante similitud. Las únicas diferencias encontradas en los datos tienen relación con el hecho de que en los ataques complejos / $\mathrm{C}+\mathrm{l} /$ se manifiestan más casos de mantención del segundo elemento; ya se ha explicado por qué ocurre el fenómeno anterior. En relación con la segunda etapa, en la cual se observa el surgimiento de un elemento consonántico en la segunda posición del ataque complejo, se concluye que la restricción *COMPLEXNCL (que milita en contra de los núcleos silábicos complejos) es la restricción de marcadez más adecuada para dar cuenta del proceso de semiconsonantización en los niños hablantes de español, ya que en esta lengua los diptongos crecientes poseen una frecuencia de aparición considerable.

Finalmente, se concluye que en la muestra estudiada no es posible afirmar que la dominancia de las restricciones de marcadez sobre las de fidelidad es exclusiva de los primeros grupos de edad. Sin embargo, sí es acertado concluir que la dominancia de las restricciones de marcadez tiene lugar en los primeros estadios de adquisición de cada sujeto. 


\section{Referencias bibliográficas}

Archangeli, D. (1999). Introducing Optimality Theory. Annual Review of Anthropology, (28), 531-552.

Barlow, J. (2005a). Phonological change and the representation of consonant clusters in Spanish: A case study. Clinical Linguistics \& Phonetics, (19), 659-679.

(2005b). Sonority effects in the production of consonant clusters by Spanish-speaking children. En Eddington, D. (Eds.), Selected proceedings from the 6th Conference on the Acquisition of Spanish and Portuguese as First and Second Languages (pp. 1-14). Somerville, MA: Cascadilla Proceedings Project.

Boersma, P. \& Levelt, C. (2003). Optimality Theory and phonological acquisition. Annual Review of Language Acquisition, (3), 1-50.

Bosch, L. (2004). Evaluación fonológica del habla infantil. Barcelona: Masson.

Cutillas, J. (2003). Universalidad y especificidad de las restricciones fonológicas: Acento y Fonotaxis en inglés. (Tesis doctoral). Universidad de Murcia, Murcia. España.

Clements, G. \& Keyser, S. (1983). CV Phonology: A theory of Syllable. Cambridge: The MIT Press.

Chin, S. (1999). The role of the sonority hierarchy in delayed phonological systems. En T. W. Powell (Eds.), Pathologies of speech and language: Contributions of clinical phonetics and linguistics (pp. 109-117). New Orleans, EE.UU.: International Clinical Phonetics and Linguistics Association.

D'Introno, F., Del Teso, E. \& Weston, R. (1995). Fonética y Fonología actual del Español. Madrid: Cátedra Ediciones.

Gierut, J. (1999). Syllable onsets: clusters and adjuncts in acquisition. Journal of Speech, Language and Hearing Research, (42), 708-726. 
Gili Gaya, S. (1966). Elementos de fonética general (pp. 117-118). Madrid: Gredos.

Gnanadesikan, A. (2004). Markedness and faithfulness constraints in child phonology. In R. Kager, J. Pater and W. Zonneveld (eds), Constraints in Phonological Acquisition. Cambridge: CUP:73-108.

González, M. (1989). Análisis del desarrollo fonológico en sujetos malagueños. Infancia y aprendizaje, (48), 7-24.

Kehoe, M., Hilaire-Debove, G., Demuth, K., \& Lleó, C. (2008). The structure of branching onsets and rising diphthongs: Evidence from the acquisition of French and Spanish. Language Acquisition, (15), 5-57.

Levelt, Schiller \& Levelt, (1999/2000). The Acquisition of Syllable Types. Language Acquisition, 8 (3), 237-264.

Núñez, R. \& Morales-Front, A. (1999). Fonología Generativa contemporánea de la lengua española. Washington: Georgetown University Press.

Ohala, D. (1999). The influence of sonority on children's cluster reductions, Journal of Communication Disorders, (32), 397-422.

Pater, J. (1997). Minimal violation and phonological development. Language Acquisition, 6 (3), 201-253.

\& Barlow, J. (2003). Constraint conflict in cluster reduction. Journal of Child Language (30), 487-526.

Prince, A. \& Smolensky, P. (1993). Optimality Theory: Constraints interaction in generative grammar. Manuscrito, Universidad de Rutgers y Universidad de Colorado. ROA-537.

Stampe, D. (1969). A Dissertation on Natural Phonology. New York: Garland Press.

Vivar, P. \& León, H. (2009). Desarrollo fonológico-fonético en un grupo de niños entre 3 y 5, 11 años. CEFAC, 11 (2), 190-198. 
